\title{
Global Estimates for Mixed Methods for Second Order Elliptic Equations
}

\author{
By Jim Douglas, Jr., and Jean E. Roberts
}

\begin{abstract}
Global error estimates in $L^{2}(\Omega), L^{\infty}(\Omega)$, and $H^{-s}(\Omega), \Omega$ in $\mathbf{R}^{2}$ or $\mathbf{R}^{3}$, are derived for a mixed finite element method for the Dirichlet problem for the elliptic operator $L p=$ $-\operatorname{div}(a \operatorname{grad} p+\mathbf{b} p)+c p$ based on the Raviart-Thomas-Nedelec space $\mathbf{V}_{h} \times W_{h} \subset$ $\mathbf{H}(\operatorname{div} ; \Omega) \times L^{2}(\Omega)$. Optimal order estimates are obtained for the approximation of $p$ and the associated velocity field $\mathbf{u}=-(a$ grad $p+\mathbf{b} p)$ in $L^{2}(\Omega)$ and $H^{-s}(\Omega), 0 \leqslant s \leqslant k+1$, and, if $\Omega \subset \mathbf{R}^{2}$, for $p$ in $L^{\infty}(\Omega)$.
\end{abstract}

1. Introduction. Let $\Omega$ be a bounded domain in $\mathbf{R}^{2}$ or $\mathbf{R}^{3}$ with boundary $\partial \Omega$, and assume that the Dirichlet problem

$$
\begin{aligned}
& \text { (a) } L p=-\operatorname{div}(a(x) \operatorname{grad} p+\mathbf{b}(x) p)+c(x) p=f(x), \quad x \in \Omega, \\
& \text { (b) } p=-g(x), \quad x \in \partial \Omega,
\end{aligned}
$$

is solvable for $\{f, g\} \in L^{2}(\Omega) \times H^{3 / 2}(\partial \Omega)$ and that

$$
\|p\|_{2} \leqslant Q\left\{\|f\|_{0}+|g|_{3 / 2}\right\}
$$

where we shall indicate the norms in $W^{m, q}(\Omega)$ by $\|\cdot\|_{m, q}$ with $q=2$ being omitted and in $W^{m, q}(\partial \Omega)$ by $|\cdot|_{m, q}$, respectively, again with $q=2$ being omitted. Let

$$
\mathbf{u}=-(a \operatorname{grad} p+\mathbf{b} p) \text {, }
$$

and set

$$
\alpha(x)=a(x)^{-1}, \quad \beta(x)=\alpha(x) \mathbf{b}(x) .
$$

Then, the differential equation (1.1a) can be written in the form of the first order system

$$
\text { (a) } \alpha \mathbf{u}+\operatorname{grad} p+\beta p=\mathbf{0} \text {, }
$$$$
\text { (b) } \operatorname{div} \mathbf{u}+c p=f, \quad x \in \Omega \text {. }
$$

Let

$$
\begin{aligned}
& \text { (a) } \mathbf{V}=\mathbf{H}(\operatorname{div} ; \Omega)=\left\{\mathbf{u} \in L^{2}(\Omega)^{2}: \operatorname{div} \mathbf{u} \in L^{2}(\Omega)\right\} \text {, } \\
& \text { (b) } W=L^{2}(\Omega) .
\end{aligned}
$$

Then, the weak form of (1.1) that we shall treat is given by seeking a pair $\{\mathbf{u}, p\} \in \mathbf{V} \times W$ such that

$$
\begin{aligned}
& \text { (a) }(\alpha \mathbf{u}, \mathbf{v})-(\operatorname{div} \mathbf{v}, p)+(\boldsymbol{\beta} p, \mathbf{v})=\langle g, \mathbf{v} \cdot v\rangle, \quad \mathbf{v} \in \mathbf{v}, \\
& \text { (b) }(\operatorname{div} \mathbf{u}, w)+(c p, w)=(f, w), \quad w \in W,
\end{aligned}
$$

Received April 25, 1983; revised April 10, 1984.

1980 Mathematics Subject Classification. Primary 65N30. 
where $v$ is the outer normal to $\partial \Omega$ and the inner product in $L^{2}(\Omega)^{n}$ is indicated by $(\cdot, \cdot)$ and in $L^{2}(\partial \Omega)$ by $\langle\cdot, \cdot\rangle$; the same notations will be used for the dualities between $H^{s}(\Omega)$ or $H_{0}^{s}(\Omega)$ and its dual and $H^{-s}(\partial \Omega)$ and $H_{0}^{-s}(\partial \Omega)$, respectively. It is known [13] that $\mathbf{v} \cdot v \in H^{-1 / 2}(\partial \Omega)$ when $\mathbf{v} \in \mathbf{V}$.

Let

$$
\mathbf{V}_{h} \times W_{h}=\mathbf{V}\left(\Omega, \mathscr{T}_{h}, k\right) \times W\left(\Omega, \mathscr{T}_{h}, k\right)
$$

denote the Raviart-Thomas-Nedelec space [7], [8], [13] of index $k$ associated with a quasi-regular partition $\mathscr{T}_{h}$ of $\Omega$ into triangles and rectangles (or parallelograms) of diameter not greater than $h$ such that every angle of each triangle is bounded below by a positive constant. Boundary triangles or rectangles are allowed to have one curvilinear edge. Let $\Omega \subset \mathbf{R}^{2}$ for the moment and let $P_{k}(T)$ denote the restriction of the polynomials of total degree $k$ to the set $T$; similarly, let $Q_{k}(T)$ indicate the restriction of $P_{k} \otimes P_{k}$ to $T$. Also, let $\mathbf{P}_{k}(T)=P_{k}(T)^{2}$. If $T \in \mathscr{T}_{h}$ is a triangle, let

$$
\mathbf{V}(T)=\mathbf{P}_{k}(T) \oplus \operatorname{Span}\left(\mathbf{x} P_{k}(T)\right), \quad W(T)=P_{k}(T)
$$

Similarly, if $T$ is a rectangle, let

$$
\mathbf{V}(T)=\left\{\mathbf{v} \in \mathbf{Q}_{k+1}(T): \partial v_{i}^{k+1} / \partial x_{j}^{k+1}=0 j \neq i\right\}, \quad W(T)=Q_{k}(T) .
$$

Then, let

$$
\begin{aligned}
\mathbf{V}_{h} & =\mathbf{V}\left(k, \mathscr{T}_{h}\right)=\left\{\mathbf{v} \in \mathbf{H}(\operatorname{div}, \Omega):\left.\mathbf{v}\right|_{T} \in \mathbf{V}(T), T \in \mathscr{T}_{h}\right\}, \\
W_{h} & =W\left(k, \mathscr{T}_{h}\right)=\left\{w \in L^{2}(\Omega):\left.w\right|_{T} \in W(T), T \in \mathscr{T}_{h}\right\} .
\end{aligned}
$$

The requirement that $\mathbf{v} \in \mathbf{H}(\operatorname{div}, \Omega)$ is equivalent to asking for continuity in the normal component across the edges of the elements; i.e., if $e=\bar{T}_{i} \cap \bar{T}_{j}$ and $v_{i}$ is the outer normal to $T_{i}$ on $e$, then $\left.\mathbf{v}\right|_{T_{i}} \cdot v_{i}+\left.\mathbf{v}\right|_{T_{j}} \cdot v_{j}=0$ on $e$. The above formulation of the Raviart-Thomas spaces coincides with that of Raviart and Thomas [8] for rectangular elements and is the modification due to Nedelec [7] on triangular elements. With the obvious modification that $\mathbf{P}_{k}(T)$ indicate $P_{k}(T)^{3}$ for $T$ being a simplex in $\mathbf{R}^{3}$, etc., the spaces $\mathbf{V}_{h} \times W_{h}$ defined above remain valid for $\Omega \subset \mathbf{R}^{3}$; consequently, the method to be specified in (1.8) is applicable for problems in three space variables.

Our mixed method for approximating the solution of (1.1) is defined by the determining of a pair $\left\{\mathbf{u}_{h}, p_{h}\right\} \in \mathbf{V}_{h} \times W_{h}$ such that

$$
\begin{aligned}
& \text { (a) }\left(\alpha \mathbf{u}_{h}, \mathbf{v}\right)-\left(\operatorname{div} \mathbf{v}, p_{h}\right)+\left(\beta p_{h}, \mathbf{v}\right)=\langle g, \mathbf{v} \cdot v\rangle, \quad \mathbf{v} \in \mathbf{V}_{h}, \\
& \text { (b) }\left(\operatorname{div} \mathbf{u}_{h}, w\right)+\left(c p_{h}, w\right)=(f, w), \quad w \in W_{h} .
\end{aligned}
$$

This procedure, which was given explicitly by the authors in [2], represents the simplest and most direct extension of the standard mixed method [1], [4], [5], [8], [10], [13].

We shall establish the existence and uniqueness of a solution of (1.8) for sufficiently small $h$; moreover, we shall show that the differences $p-p_{h}$ and $\mathbf{u}-\mathbf{u}_{h}$ are of optimal order in $L^{2}(\Omega)$ and in $H^{-s}(\Omega)$ or $H^{s}(\Omega)^{\prime}$ for $s \leqslant k+1$, provided, of course, that the domain $\Omega$ and the solution $p$ are sufficiently regular. Indeed, we shall prove that

$$
\begin{aligned}
& \text { (a) }\left\|p-p_{h}\right\|_{0} \leqslant Q\|p\|_{k+1} h^{k+1}, \quad\left\|\mathbf{u}-\mathbf{u}_{h}\right\|_{0} \leqslant Q\|p\|_{k+2} h^{k+1}, \\
& \text { (b) }\left\|p-p_{h}\right\|_{-k-1}+\left\|\mathbf{u}-\mathbf{u}_{h}\right\|_{-k-1} \leqslant Q\|p\|_{k+3} h^{2 k+2}
\end{aligned}
$$


We shall show as a trivial corollary of our basic duality lemma that, when $\Omega \subset \mathbf{R}^{2}$,

$$
\left\|p-p_{h}\right\|_{0, \infty} \leqslant Q\left\{\|p\|_{k+1, \infty}+\|p\|_{k+2}\right\} h^{k+1},
$$

a result obtained earlier by Scholz [10] for the Laplace operator; he has since [11], [12] found a better result for the Laplace operator than (1.10).

An entirely analogous development can be given for the Dirichlet problem

$$
\begin{aligned}
& \text { (a) }-\operatorname{div}(a(x) \cdot \operatorname{grad} p)+\mathbf{b}(x) \cdot \operatorname{grad} p+c(x) p=f(x), \quad x \in \Omega, \\
& \text { (b) } p=-g(x), \quad x \in \partial \Omega \text {. }
\end{aligned}
$$

In this case the auxiliary variable is chosen to be

$$
\mathbf{v}=-a \operatorname{grad} p
$$

see [2] for the corresponding $L^{2}(\Omega)$-estimates for the error. The basic arguments of this paper can be applied to obtain negative norm and $L^{\infty}(\Omega)$ estimates of the same nature as those we derive.

The extension of our convergence results to the case $\Omega \subset \mathbf{R}^{3}$ will be noted in the last section of the paper. Until then, consider $\Omega$ to be a planar domain.

2. The Raviart-Thomas Projections and the Error Equation. The Raviart-Thomas spaces that we use consist on each triangle (we include "rectangle" as a special meaning of "triangle") of exactly the sets of polynomials that would have resulted on the triangle when all of its edges are linear; i.e., the space is unmodified on boundary triangles. Thus, we preserve the relation

$$
\operatorname{div} \mathbf{V}_{h}=W_{h} .
$$

For polygonal domains Raviart and Thomas [8] defined a projection

$$
\Pi_{h} \times P_{h}: \mathbf{V} \times W \rightarrow \mathbf{V}_{h} \times W_{h}
$$

having the properties:

(i) $P_{h}$ is $L^{2}(\Omega)$-projection;

(ii) the following diagram commutes:

$$
\begin{array}{ccccc}
\mathbf{V} & \stackrel{\operatorname{div}}{\rightarrow} & W & & \\
\Pi_{h} \downarrow & & \downarrow P_{h} & & \\
\mathbf{V}_{h} & \stackrel{\operatorname{div}}{\rightarrow} & W_{h} & \rightarrow & 0 ;
\end{array}
$$

i.e., $\operatorname{div} \Pi_{h}=P_{h} \operatorname{div}: \mathrm{V} \stackrel{\text { onto }}{\rightarrow} W_{h}$;

(iii) the following approximation properties hold:

$$
\text { (a) }\left\|\mathbf{u}-\Pi_{h} \mathbf{u}\right\|_{0} \leqslant Q\|\mathbf{u}\|_{r} h^{r}, \quad 1 \leqslant r \leqslant k+1,
$$

$$
\begin{aligned}
& \text { (b) }\left\|\operatorname{div}\left(\mathbf{u}-\Pi_{h} \mathbf{u}\right)\right\|_{-s} \leqslant Q\|\operatorname{div} \mathbf{u}\|_{r} h^{r+s}, \quad 0 \leqslant r, s \leqslant k+1, \\
& \text { (c) }\left\|p-P_{h} p\right\|_{-s} \leqslant Q\|p\|_{r} h^{r+s}, \quad 0 \leqslant r, s \leqslant k+1 ;
\end{aligned}
$$

(iv) (redundant)

$$
\begin{aligned}
& \text { (a) }\left(\operatorname{div}\left(\mathbf{u}-\Pi_{h} \mathbf{u}\right), w_{h}\right)=0, \quad w_{h} \in W_{h}, \\
& \text { (b) }\left(\operatorname{div} \mathbf{v}_{h}, p-P_{h} p\right)=0, \quad \mathbf{v}_{h} \in \mathbf{V}_{h} .
\end{aligned}
$$


In the next to last section of this paper, Douglas N. Arnold and we present a way to modify the definition $\Pi_{h}$ restricted to a boundary triangle having a curved edge so that all of the above requirements are met when the resulting local projection is combined with the standard local version.

Let

$$
\begin{aligned}
& \text { (a) } \boldsymbol{\xi}=\mathbf{u}-\mathbf{u}_{h}, \quad \boldsymbol{\sigma}=\Pi_{h} \mathbf{u}-\mathbf{u}_{h}, \\
& \text { (b) } \eta=p-p_{h}, \quad \tau=P_{h} p-p_{h}, \quad \rho=p-P_{h} p .
\end{aligned}
$$

It then follows immediately from (1.7) and (1.8) that

$$
\begin{aligned}
& \text { (a) }(\alpha \xi, \mathbf{v})-(\operatorname{div} \mathbf{v}, \eta)+(\beta \eta, \mathbf{v})=0, \quad \mathbf{v} \in \mathbf{V}_{h}, \\
& \text { (b) }(\operatorname{div} \xi, w)+(c \eta, w)=0, \quad w \in W_{h},
\end{aligned}
$$

or, equivalently, that, as a result of $(2.4 \mathrm{~b})$,

(a) $(\alpha \boldsymbol{\xi}, \mathbf{v})-(\operatorname{div} \mathbf{v}, \tau)+(\beta \tau, \mathbf{v})=-(\boldsymbol{\beta} \rho, \mathbf{v}), \quad \mathbf{v} \in \mathbf{V}_{h}$,

(b) $(\operatorname{div} \xi, w)+(c \tau, w)=-(c \rho, w), \quad w \in W_{h}$.

The argument to be presented below is a refinement and extension of that given briefly by the authors [2]. As such, it also represents an extension of some results of Johnson and Thomée [5] and is a natural analogue of the argument developed by Schatz [9] for treating Galerkin methods for the Dirichlet problem for noncoercive operators. We shall first demonstrate several forms of a duality lemma and then derive error estimates.

The orthogonalities given in (2.4) play a fundamental role in our discussion of duality. Elsewhere, Arnold, Chaitan Gupta, and Douglas treat the plane elasticity problem in a similar fashion using a mixed method based on a new family of elements designed to satisfy relations corresponding to (2.4) and having approximation properties of a somewhat more complicated nature than those of (2.3). Arnold, Brezzi, and Douglas will discuss a yet different approach to plane elasticity based on a set of finite elements which do not satisfy the usual symmetry properties for the stress tensor but do satisfy (2.4). Also, Douglas and Milner have developed interior and superconvergence results for the mixed method (1.8).

3. The Duality Lemmas. We shall employ duality with respect to $H^{s}(\Omega)$ in place of $H_{0}^{s}(\Omega)$; i.e., if $\varphi \in L^{2}(\Omega)$, then

$$
\|\varphi\|_{-s}=\|\varphi\|_{-s, 2, \Omega}=\sup _{0 \neq \psi \in H^{s}(\Omega)} \frac{(\varphi, \psi)}{\|\psi\|_{s}}, \quad s \geqslant 0 .
$$

Nothing of interest would change if the usual dual space $H^{-s}(\Omega)=\left(H_{0}^{s}(\Omega)\right)^{\prime}$ is used. We shall say that $\Omega$ is $(s+2)$-regular if the Dirichlet problem

(a) $L^{*} \varphi=\psi, \quad x \in \Omega$,

(b) $\varphi=0, \quad x \in \partial \Omega$,

is uniquely solvable for $\psi \in L^{2}(\Omega)$ and if

$$
\|\varphi\|_{s+2} \leqslant Q\|\psi\|_{s}
$$

for all $\psi \in H^{s}(\Omega)$. 
If $f \in \mathbf{V}^{\prime}$, the dual space of $\mathbf{V}$, then it can be represented by a pair $\left\{\mathbf{f}_{0}, f_{1}\right\}$ of functions such that $\mathrm{f}_{0} \in L^{2}(\Omega)^{2}, f_{1} \in L^{2}(\Omega)$, and

$$
f(\mathbf{v})=\left(\mathbf{f}_{0}, \mathbf{v}\right)+\left(f_{1}, \operatorname{div} \mathbf{v}\right), \quad \mathbf{v} \in \mathbf{v} .
$$

The first version of our duality lemma is as follows.

LEMMA 3.1. Let the index $k$ of $\mathbf{V}_{h} \times W_{h}$ be at least one and let $0 \leqslant s \leqslant k-1$. Assume that $\Omega$ is $(s+2)$-regular. Let $\zeta \in \mathbf{V}, f=\left\{\mathbf{f}_{0}, f_{1}\right\} \in \mathbf{V}^{\prime}$, and $g \in W^{\prime}=L^{2}(\Omega)$. If $z \in W_{h}$ satisfies the relations

(a) $(\alpha \zeta, \mathbf{v})-(\operatorname{div} \mathbf{v}, z)+(\boldsymbol{\beta} z, \mathbf{v})=f(\mathbf{v}), \quad \mathbf{v} \in \mathbf{V}_{h}$,

(b) $(\operatorname{div} \zeta, w)+(c z, w)=g(w), \quad w \in W_{h}$,

then, for sufficiently small $h$,

$$
\begin{aligned}
\|z\|_{-s} \leqslant Q\left\{h^{s+1}\|\zeta\|_{0}+h^{s+2}\|\operatorname{div} \zeta\|_{0}+\left\|\mathbf{f}_{0}\right\|_{-s-1}+h^{s+1}\left\|f_{0}\right\|_{0}\right. \\
\left.+\left\|f_{1}\right\|_{-s}+h^{s}\left\|f_{1}\right\|_{0}+\|g\|_{-s-2}+h^{s+2}\|g\|_{0}\right\} .
\end{aligned}
$$

Proof. Let $\psi \in H^{s}(\Omega)$, and let $\varphi \in H^{s+2}(\Omega) \cap H_{0}^{1}(\Omega)$ be the solution of (3.2). By (3.5a) and (2.4a),

$$
\begin{aligned}
(z, \psi)= & (z,-\operatorname{div}(a \operatorname{grad} \varphi)+\beta \cdot a \operatorname{grad} \varphi+c \varphi) \\
= & -\left(\alpha \zeta, \Pi_{h}(a \operatorname{grad} \varphi)\right)+f\left(\Pi_{h}(a \operatorname{grad} \varphi)\right) \\
& +\left(\beta z, a \operatorname{grad} \varphi-\Pi_{h}(a \operatorname{grad} \varphi)\right)+(c z, \varphi) .
\end{aligned}
$$

Then, by (3.5b),

$$
(c z, \varphi)=\left(c z+\operatorname{div} \zeta, \varphi-P_{h} \varphi\right)+g\left(P_{h} \varphi\right)-(\operatorname{div} \zeta, \varphi)
$$

and

$$
\begin{aligned}
-(\operatorname{div} \zeta, \varphi) & =(\zeta, \operatorname{grad} \varphi)=(\alpha \zeta, a \operatorname{grad} \varphi) \\
& =\left(\alpha \zeta, \Pi_{h}(a \operatorname{grad} \varphi)\right)+\left(\alpha \zeta, a \operatorname{grad} \varphi-\Pi_{h}(a \operatorname{grad} \varphi)\right) .
\end{aligned}
$$

These relations combine to show that

$$
\begin{aligned}
(z, \psi)= & f\left(\Pi_{h}(a \operatorname{grad} \varphi)\right)+g\left(P_{h} \varphi\right) \\
& +\left(\alpha \zeta+\beta z, a \operatorname{grad} \varphi-\Pi_{h}(a \operatorname{grad} \varphi)\right)+\left(\operatorname{div} \zeta+c z, \varphi-P_{h} \varphi\right) .
\end{aligned}
$$

Then,

$$
\begin{aligned}
\left|f\left(\Pi_{h}(a \operatorname{grad} \varphi)\right)\right| \leqslant & |f(a \operatorname{grad} \varphi)|+\left|f\left(a \operatorname{grad} \varphi-\Pi_{h}(a \operatorname{grad} \varphi)\right)\right| \\
\leqslant & \left\|f_{0}\right\|_{-s-1}\|a \operatorname{grad} \varphi\|_{s+1}+\left\|f_{1}\right\|_{-s}\|\operatorname{div}(a \operatorname{grad} \varphi)\|_{s} \\
& +\left\|f_{0}\right\|_{0}\left\|a \operatorname{grad} \varphi-\Pi_{h}(a \operatorname{grad} \varphi)\right\|_{0} \\
& +\left\|f_{1}\right\|_{0}\left\|\operatorname{div}\left(a \operatorname{grad} \varphi-\Pi_{h}(a \operatorname{grad} \varphi)\right)\right\|_{0} \\
\leqslant & Q\left\{\left\|f_{0}\right\|_{-s-1}+h^{s+1}\left\|f_{0}\right\|_{0}+\left\|f_{1}\right\|_{-s}+h^{s}\left\|f_{1}\right\|_{0}\right\}\|\psi\|_{s},
\end{aligned}
$$

by (2.3) and (3.3). Similarly,

$$
\left|g\left(P_{h} \varphi\right)\right| \leqslant Q\left\{\|g\|_{-s-2}+h^{s+2}\|g\|_{0}\right\}\|\psi\|_{s}
$$


Again by (2.3),

$$
\begin{aligned}
\mid(\alpha \zeta+\beta z, a \operatorname{grad} \varphi- & \left.\Pi_{h}(a \operatorname{grad} \varphi)\right)|+|\left(\operatorname{div} \zeta+c z, \varphi-P_{h} \varphi\right) \mid \\
& \leqslant Q\left\{h^{s+1}\left(\|\zeta\|_{0}+\|z\|_{0}\right)+h^{s+2}\left(\|\operatorname{div} \zeta\|_{0}+\|z\|_{0}\right)\right\}\|\psi\|_{s} .
\end{aligned}
$$

Thus,

$$
\begin{aligned}
\|z\|_{-s} \leqslant Q\left\{h^{s+1}\|\zeta\|_{0}+h^{s+2}\|\operatorname{div} \zeta\|_{0}+\left\|\mathbf{f}_{0}\right\|_{-s-1}+\left\|f_{1}\right\|_{-s}+h^{s+1}\left\|\mathbf{f}_{0}\right\|_{0}\right. \\
\left.+h^{s}\left\|f_{1}\right\|_{0}+\|g\|_{-s-2}+h^{s+2}\|g\|_{0}+h^{s+1}\|z\|_{0}\right\} .
\end{aligned}
$$

Consider $s=0$. For $h$ sufficiently small, the $h\|z\|_{0}$-term on the right-hand side can be absorbed into the left-hand side, and the inequality (3.6) has been established for $s=0$. Then, for $0<s \leqslant k-1$, (3.6) holds, provided that $\|g\|_{-2} h^{s+1}$ can be bounded by the terms on the right-hand side of (3.6). But, standard interpolation theory for the spaces $H^{s}(\Omega)^{\prime}[6]$ implies that

$$
\|g\|_{-2} \leqslant Q\|g\|_{0}^{s /(s+2)}\|g\|_{-s-2}^{2 /(s+2)} \leqslant Q\left\{h\|g\|_{0}+h^{-s / 2}\|g\|_{-s-2}\right\}
$$

and the proof of the lemma has been completed.

The cases $k=0$ and $s=k$ when $k \geqslant 1$ are not covered by Lemma 3.1. Because of reaching the limits of approximability provided by (2.3), a change in the form of the estimate occurs. In the applications below to finding error estimates, we shall be able to maintain optimal order estimates when the $s=k$ lemma is used, but a cost of additional regularity results.

LEMMA 3.2. Let the index $k$ of $\dot{V}_{h} \times W_{h}$ be nonnegative, and let $\Omega$ be $(k+2)$-regular. Let $\zeta \in \mathbf{V}, f=\left\{\mathbf{f}_{0}, 0\right\} \in \mathbf{V}^{\prime}$, and $g \in L^{2}(\Omega)$. If $z \in W_{h}$ satisfies (3.5), then for $h$ sufficiently small,

$$
\|z\|_{-k} \leqslant Q\left\{h^{k+1}\left(\|\zeta\|_{0}+\|\operatorname{div} \zeta\|_{0}+\left\|\mathbf{f}_{0}\right\|_{0}+\|g\|_{0}\right)+\left\|\mathbf{f}_{0}\right\|_{-k-1}+\|g\|_{-k-2}\right\} .
$$

Proof. The relation (3.7) remains valid, except that $f_{1}$ has been set to zero. Now, the limit on approximability for $\varphi-P_{h} \varphi$ reduces the exponent $s+2=k+2$ to $k+1$ in two places in the arguments; otherwise, the proof is unchanged.

It should be noted that all factors of $h$ occurring in the estimates (3.6) and (3.9) come from the use of (2.3). Since the projections $\Pi_{h}$ and $P_{h}$ are defined locally through moments over individual triangles (including boundary ones), these bounds can be sharpened to become more local in character, since we have assumed a minimum angle condition for the polygons $T \in \mathscr{T}_{h}$. We have actually proved, in place of (3.6), that (when, say, $f_{1}=0$ and $\operatorname{diam}(T)=h_{T}$ ),

$$
\begin{array}{r}
\|z\|_{-s}^{2} \leqslant Q\left\{\sum_{T \in \mathscr{T}_{h}}\left[h_{T}^{2 s+2}\left(\|\xi\|_{0, T}^{2}+\left\|\mathbf{f}_{0}\right\|_{0, T}^{2}\right)+h_{T}^{2 s+4}\left(\|\operatorname{div} \zeta\|_{0, T}^{2}+\|g\|_{0, T}^{2}\right)\right]\right. \\
\left.+\left\|\mathbf{f}_{0}\right\|_{-s-1}^{2}+\|g\|_{-s-2}^{2}\right\}
\end{array}
$$

for $0 \leqslant s \leqslant k-1$. We shall not pursue these additional refinements.

4. Error Estimates in $L^{2}(\Omega)$. Assume momentarily that (1.8) has a unique solution, at least for small $h$. That it does, will be easily observed from the convergence analysis. Now, apply either Lemma 3.1 with $s=0$ or Lemma 3.2 with $k=0$ to the 
error equations in the form (2.7). Then, for $h$ small and for $\Omega$ 2-regular,

$$
\|\tau\|_{0} \leqslant Q\left\{h\|\xi\|_{0}+h^{2-\delta_{k 0}}\|\operatorname{div} \xi\|_{0}+\|\rho\|_{-1}+h\|\rho\|_{0}\right\} .
$$

Since

$$
\|\rho\|_{-1}+h\|\rho\|_{0} \leqslant Q\|p\|_{r} h^{r+1}
$$

for $0 \leqslant r \leqslant k+1$ by $(2.3 \mathrm{c})$,

$$
\|\tau\|_{0} \leqslant Q\left\{h\|\xi\|_{0}+h^{2-\delta_{k 0}}\|\operatorname{div} \xi\|_{0}+\|p\|_{r} h^{r+1}\right\}
$$

for $0 \leqslant r \leqslant k+1$, and

$$
\|\eta\|_{0} \leqslant Q\left\{\|\boldsymbol{\xi}\|_{0} h+h^{2-\delta_{k 0}}\|\operatorname{div} \xi\|_{0}+\|p\|_{r} h^{r}\right\}, \quad 0 \leqslant r \leqslant k+1 .
$$

Since, by $(2.4 \mathrm{a}),(\operatorname{div} \sigma, w)=(\operatorname{div} \xi, w)$ for $w \in W_{h}$, it follows from $(2.6 \mathrm{~b})$ and the choice $w=\operatorname{div} \sigma \in W_{h}$ that

$$
\|\operatorname{div} \boldsymbol{\sigma}\|_{0} \leqslant Q\|\eta\|_{0}
$$

so that

$$
\|\operatorname{div} \boldsymbol{\xi}\|_{0} \leqslant Q\left\{\|\eta\|_{0}+\|\operatorname{div} \mathbf{u}\|_{q} h^{q}\right\}, \quad 0 \leqslant q \leqslant k+1 .
$$

Next, take the test function $\mathbf{v}=\boldsymbol{\sigma}$ in (2.6a) to see that

$$
(\alpha \boldsymbol{\sigma}, \boldsymbol{\sigma})=(\operatorname{div} \boldsymbol{\sigma}, \eta)-(\beta \eta, \boldsymbol{\sigma})-\left(\alpha\left(\mathbf{u}-\Pi_{h} \mathbf{u}\right), \boldsymbol{\sigma}\right) ;
$$

consequently,

$$
\widetilde{\| \boldsymbol{\sigma}} \|_{0} \leqslant Q\left\{\|\eta\|_{0}+\|\mathbf{u}\|_{t} h^{t}\right\}, \quad 1 \leqslant t \leqslant k+1
$$

and

$$
\|\boldsymbol{\xi}\|_{0} \leqslant Q\left\{\|\eta\|_{0}+\|\mathbf{u}\|_{t} h^{t}\right\}, \quad 1 \leqslant t \leqslant k+1 .
$$

If (4.4) and (4.6) are substituted into (4.2), then for $0 \leqslant r \leqslant k+1,0 \leqslant q \leqslant k+1$, and $1 \leqslant t \leqslant k+1$ it follows that

$$
\|\eta\|_{0} \leqslant Q\left\{h\|\eta\|_{0}+\|p\|_{r} h^{r}+\|\mathbf{u}\|_{t} h^{t+1}+\|\operatorname{div} \mathbf{u}\|_{q} h^{q+2-\delta_{k 0}}\right\} \text {. }
$$

Thus, for small $h$ and the choices $r=t+1=q+2-\delta_{k 0}$,

$$
\|\eta\|_{0} \leqslant \begin{cases}Q\|p\|_{2} h & \text { if } k=0, \\ Q\|p\|_{r} h^{r} & \text { if } k \geqslant 1 \text { and } 2 \leqslant r \leqslant k+1\end{cases}
$$

since $\|\mathbf{u}\|_{r-1}+\|\operatorname{div} \mathbf{u}\|_{r-2} \leqslant Q\|p\|_{r}$. The estimates can be written in a more compact form for $k \geqslant 0$ and $0 \leqslant q \leqslant 2$ as

$$
\|\eta\|_{0} \leqslant Q\|p\|_{r+q} h^{r}, \quad 2-q \leqslant r \leqslant k+1 .
$$

It then follows immediately that, for $k \geqslant 0$ and $0 \leqslant q \leqslant 1$,

$$
\begin{aligned}
& \text { (a) }\|\xi\|_{0} \leqslant Q\|p\|_{r+1+q} h^{r}, \quad 1-q \leqslant r \leqslant k+1, \\
& \text { (b) }\|\operatorname{div} \xi\|_{0} \leqslant Q\|p\|_{r+2} h^{r}, \quad 0 \leqslant r \leqslant k+1,
\end{aligned}
$$

and the analysis of the errors, as measured in $L^{2}(\Omega)$, is finished.

Before collecting the results of the error analysis in a theorem, let us demonstrate the existence and uniqueness of the solution of (1.8). Since (1.8) is linear, it suffices to establish uniqueness; thus, we suppose the data functions $f$ and $g$ to vanish. The choice $w=\operatorname{div} \mathbf{u}_{h}$ in $(1.8 \mathrm{~b})$ implies that

$$
\left\|\operatorname{div} \mathbf{u}_{h}\right\|_{0} \leqslant Q\left\|p_{h}\right\|_{0} .
$$


Next, note that Lemma 3.1 or Lemma 3.2 implies that

$$
\left\|p_{h}\right\|_{0} \leqslant Q\left\{h\left\|\mathbf{u}_{h}\right\|_{0}+h\left\|\operatorname{div} \mathbf{u}_{h}\right\|_{0}\right\}
$$

so that, for small $h$,

$$
\left\|p_{h}\right\|_{0} \leqslant Q h\left\|\mathbf{u}_{h}\right\|_{0} .
$$

If the test function $\mathbf{v}$ in (1.8a) is taken to be $\mathbf{u}_{h}$, then it follows that

$$
\left\|\mathbf{u}_{h}\right\|_{0} \leqslant Q\left\|p_{h}\right\|_{0} \leqslant Q h\left\|\mathbf{u}_{h}\right\|_{0},
$$

so that $\mathbf{u}_{h}$ vanishes for small $h$, along with $p_{h}$. So, uniqueness has been demonstrated. We have proved the following theorem.

THEOREM 4.1. Assume that the Dirichlet problem (1.1) has a unique solution $p \in H^{2}(\Omega)$ for every pair $\{f, g\} \in L^{2}(\Omega) \times H^{3 / 2}(\partial \Omega)$ and that $\Omega$ is 2-regular. Then, for $h$ sufficiently small there exists a unique solution $\left\{\mathbf{u}_{h}, p_{h}\right\} \in \mathbf{V}_{h} \times W_{h}$ of the mixed method equations (1.8). Moreover, the error $\left\{\mathbf{u}-\mathbf{u}_{h}, p-p_{h}\right\}$ can be estimated by the inequalities

$$
\begin{aligned}
& \text { (a) }\left\|p-p_{h}\right\|_{0} \leqslant \begin{cases}Q\|p\|_{2} h & \text { if } k=0, \\
Q\|p\|_{r} h^{r} & \text { if } k \geqslant 1 \text { and } 2 \leqslant r \leqslant k+1,\end{cases} \\
& \text { (b) }\left\|\mathbf{u}-\mathbf{u}_{h}\right\|_{0} \leqslant Q\|p\|_{r+1} h^{r} \quad \text { if } 1 \leqslant r \leqslant k+1 \\
& \text { (c) }\left\|\operatorname{div}\left(\mathbf{u}-\mathbf{u}_{h}\right)\right\|_{0} \leqslant Q\|p\|_{r+2} h^{r} \quad \text { if } 0 \leqslant r \leqslant k+1 .
\end{aligned}
$$

Theorem 4.1 was obtained by the authors in [2]; however, the proof given above is more direct, in that an unnecessary step in which Brezzi's general saddle point approximation results [1] were applied to a reduced problem has been avoided. The bound given by (4.1). above will allow us to obtain an $L^{\infty}(\Omega)$ estimate for $p-p_{h}$ very easily. It should also be noted that the weak problem (1.7) for nontrivial b and $c$ is not a compact perturbation of that for $b$ and $c$ vanishing; thus, Theorem 4.1 is not a straightforward corollary of the approach of Falk and Osborn [4], who have proved (at least implicitly) the same estimates (4.9) when $k \geqslant 1, \mathbf{b}=\mathbf{0}$, and the form is coercive.

The constraint that $h$ be sufficiently small in Theorem 4.1 results from not having assumed the original problem to be coercive. If in (1.8) we take $\mathbf{v}=\mathbf{u}_{h}$ and $w=p_{h}$, and if the two equations are then added, we see that

$$
\left(\alpha \mathbf{u}_{h}, \mathbf{u}_{h}\right)+\left(c p_{h}, p_{h}\right)+\left(\boldsymbol{\beta} p_{h}, \mathbf{u}_{h}\right)=\left(f, p_{h}\right)+\left\langle g, \mathbf{u}_{h} \cdot v\right\rangle .
$$

It is then easily seen that, if $c(x) \geqslant 0$ and

$$
b(x)^{2} \leqslant 4(1-\gamma) a(x) c(x)
$$

for some $\gamma \in(0,1)$, the algebraic equations of (1.8) are solvable; moreover, the error estimates hold without the constraint on $h$.

5. Error Estimates in $L^{\infty}(\Omega)$. In this section we shall impose the additional constraint on $\mathscr{T}_{h}$ that

$$
\operatorname{diam}(T)=h_{T} \geqslant \gamma_{0} h, \quad T \in \mathscr{T}_{h},
$$

for some $\gamma_{0} \in(0,1)$. Then it follows from (4.1) and (4.9) that

$$
\|\tau\|_{0} \leqslant Q\|p\|_{r+1+\delta_{k 0}} h^{r+1}, \quad 1 \leqslant r \leqslant k+1,
$$


and that, using (5.1) as well as the angle constraint for $\mathscr{T}_{h}$,

$$
\|\tau\|_{0, \infty} \leqslant Q h^{-1}\|\tau\|_{0} \leqslant Q\|p\|_{r+1+\delta_{k 0}} h^{r}, \quad 1 \leqslant r \leqslant k+1 .
$$

Thus, for $k \geqslant 0$,

$$
\begin{aligned}
\left\|p-p_{h}\right\|_{0, \infty} & \leqslant\left\|p-P_{h} p\right\|_{0, \infty}+\|\tau\|_{0, \infty} \\
& \leqslant Q\left\{\|p\|_{r, \infty}+\|p\|_{r+1+\delta_{k 0}}\right\} h^{r}, \quad 1 \leqslant r \leqslant k+1 .
\end{aligned}
$$

Note that (5.4) is an optimal order estimate; the regularity required of the solution of the differential problem is not minimal, since we need $p$ to belong to $H^{r+1}(\Omega)$ when $k \geqslant 1$ and to $H^{3}(\Omega)$ when $k=0$ to obtain $O\left(h^{r}\right)$ or $O(h)$, respectively, in $L^{\infty}(\Omega)$. Scholz [10] has demonstrated (5.4) for $k \geqslant 1$ when the differential operator $L$ has the form $-\Delta p+c p, c \geqslant 0$; his method of proof employed weighted $L^{2}$-norms. Recently, [11], [12] he has improved his earlier results to obtain optimal $L^{\infty}$-estimates for $p-p_{h}$ that are optimal with respect to order and regularity; he also obtained an estimate in $L^{\infty}$ for $\mathbf{u}-\mathbf{u}_{h}$ that is optimal modulo a factor of $|\log h|$. These results were presented for the Laplace operator. Johnson and Thomé [5] obtained a slightly weaker result than ours for $p-p_{h}$ by essentially the same observation as ours, again limited to $k \geqslant 1$ and the Laplace operator. Our result for $k=0$ seems to be new, even for the Laplace operator. The argument above is sharp only for $\Omega \subset \mathbf{R}^{2}$.

Our result can be summarized as follows.

THEOREM 5.1. Let $\mathscr{T}_{h}$ be quasi-regular and let (5.1) hold, and assume that $\Omega$ is 2-regular. Then, for $h$ sufficiently small,

$$
\left\|p-p_{h}\right\|_{0, \infty} \leqslant Q\left\{\|p\|_{r, \infty}+\|p\|_{r+1+\delta_{k 0}}\right\} h^{r}
$$

for $1 \leqslant r \leqslant k+1$.

6. Error Estimates in $H^{s}(\Omega)^{\prime}$. Let $\Omega$ be $(s+2)$-regular, and consider first the range $0 \leqslant s \leqslant k-1$ (which requires that $k \geqslant 1$ ). Then the application of Lemma 3.1 and (2.3c) to the error equations (2.7) produces the bound

$$
\|\tau\|_{-s} \leqslant Q\left\{h^{s+1}\|\boldsymbol{\xi}\|_{0}+h^{s+2}\|\operatorname{div} \xi\|_{0}+h^{r+s+1}\|p\|_{r}\right\}
$$

for $0 \leqslant r \leqslant k+1$. It then follows from Theorem 4.1 that

$$
\|\tau\|_{-s} \leqslant Q\|p\|_{r+1} h^{r+s+1}, \quad 1 \leqslant r \leqslant k+1
$$

from which we see that

$$
\|\eta\|_{-s} \leqslant Q\|p\|_{r} h^{r+s}, \quad \text { if } 2 \leqslant r \leqslant k+1 \text { and } 0 \leqslant s \leqslant k-1 .
$$

The estimate (6.2) is optimal with respect to regularity and is the desired result over the range of $s$ admitted.

We can also employ Lemma 3.2, now for $k \geqslant 0$ and $s=k$. Then, using Theorem 4.1 again,

$$
\begin{aligned}
\|\tau\|_{-k} & \leqslant Q\left\{h^{k+1}\left(\|\xi\|_{0}+\|\operatorname{div} \xi\|_{0}+\left\|p-P_{h} p\right\|_{0}\right)+\left\|p-P_{h} p\right\|_{-k-1}\right\} \\
& \leqslant Q\|p\|_{r+2} h^{r+k+1}, \quad 0 \leqslant r \leqslant k+1
\end{aligned}
$$

Thus, (6.3) can be used to obtain each of the following inequalities:

$$
\|\eta\|_{-k} \leqslant Q\|p\|_{r+1} h^{r+k}, \quad 1 \leqslant r \leqslant k+1,
$$

and

$$
\|\eta\|_{-k-1} \leqslant Q\|p\|_{r+2} h^{r+k+1}, \quad 0 \leqslant r \leqslant k+1
$$


The regularity of $\Omega$ needed for (6.5) is the same as for (6.4); i.e., $(k+2)$-regular.

Note that both (6.4) and (6.5) are optimal order estimates, in that $O\left(h^{2 k+1}\right)$ and $O\left(h^{2 k+2}\right)$ bounds are obtained when $r$ is allowed to assume its maximum value $k+1$; however, one extra derivative is required of $p$ for the $s=k$ estimate and two for $s=k+1$. Consequently, these bounds are not of the nature that would be expected to result from the general stability approach of Brezzi [1] and Falk and Osborn [4].

Consider next the divergence of $\mathbf{u}-\mathbf{u}_{h}$. Let $k \geqslant 0$ and $0 \leqslant s \leqslant k+1$, and let $\varphi \in H^{s}(\Omega)$. Then, by (2.6b),

$$
\begin{aligned}
(\operatorname{div} \xi, \varphi) & =\left(\operatorname{div} \xi, P_{h} \varphi\right)+\left(\operatorname{div} \xi, \varphi-P_{h} \varphi\right) \\
& =-(c \eta, \varphi)+\left(c \eta+\operatorname{div} \xi, \varphi-P_{h} \varphi\right),
\end{aligned}
$$

so that

$$
\|\operatorname{div} \boldsymbol{\xi}\|_{-s} \leqslant Q\left\{\|\eta\|_{-s}+h^{s}\left(\|\eta\|_{0}+\|\operatorname{div} \xi\|_{0}\right)\right\} \leqslant Q\|p\|_{r+2} h^{r+s}
$$

for $0 \leqslant r \leqslant k+1$, by Thenrem 4.1 and (6.2), (6.4), or (6.5). This estimate is optimal with respect to both rate and regularity for all admitted choices of $r$ and $s$.

Finally, let us consider bounding $\xi$ in $H^{s}(\Omega)^{\prime}$. Let $\psi \in H^{s}(\Omega)^{2}$. We should like to have a function $\varphi \in H^{s+1}(\Omega)$ such that $\psi=-a \operatorname{grad} \varphi$ and $\varphi=0$ on $\partial \Omega$; unfortunately, this overspecifies $\varphi$, so that a new argument must be given. Let $\varphi \in$ $H^{s+1}(\Omega) \cap H_{0}^{1}(\Omega)$ be the solution of the Dirichlet problem

(a) $-\operatorname{div}(a \operatorname{grad} \varphi)=\operatorname{div} \psi, \quad x \in \Omega$,

(b) $\varphi=0, \quad x \in \partial \Omega$.

Assume that $\Omega$ is $(s+1)$-regular for (6.7); this is no more a requirement on $\Omega$ than $(s+1)$-regularity for $L^{*}$. Then,

$$
\|\varphi\|_{s+1} \leqslant Q\|\operatorname{div} \psi\|_{s-1} \leqslant Q\|\psi\|_{s} .
$$

Furthermore, $\psi=-a \operatorname{grad} \varphi+\delta$, where $\operatorname{div} \delta=0$ and

$$
\|\boldsymbol{\delta}\|_{s} \leqslant Q\|\psi\|_{s} .
$$

Now,

$$
(\alpha \xi, \psi)=-(\alpha \xi, a \operatorname{grad} \varphi)+(\alpha \xi, \delta)=(\operatorname{div} \xi, \varphi)+(\alpha \xi, \delta) .
$$

As in the argument for (6.6),

$$
|(\operatorname{div} \xi, \varphi)| \leqslant Q\left\{\|\eta\|_{-s-1}+\left(\|\eta\|_{0}+\|\operatorname{div} \xi\|_{0}\right) h^{s+1}\right\}\|\psi\|_{s}
$$

for $0 \leqslant s \leqslant k$. Hence,

$$
|(\operatorname{div} \xi, \varphi)| \leqslant Q\|p\|_{r+2} h^{r+s+1}\|\psi\|_{s}, \quad 0 \leqslant r \leqslant k+1 .
$$

Then, since $\operatorname{div} \delta=0$ and by (2.4),

$$
\begin{aligned}
(\alpha \xi, \delta)= & \left(\alpha \xi, \Pi_{h} \delta\right)+\left(\alpha \xi, \delta-\Pi_{h} \boldsymbol{\delta}\right) \\
= & \left(\operatorname{div} \Pi_{h} \delta, \eta\right)-\left(\beta \eta, \Pi_{h} \delta\right)+\left(\alpha \xi, \delta-\Pi_{h} \delta\right) \\
= & \left(\operatorname{div}\left(\Pi_{h} \delta-\delta\right), \tau\right)+\left(\operatorname{div} \Pi_{h} \delta, \rho\right) \\
& -(\beta \eta, \delta)+\left(\beta \eta+\alpha \xi, \delta-\Pi_{h} \delta\right) \\
= & -(\beta \eta, \delta)+\left(\beta \eta+\alpha \xi, \delta-\Pi_{h} \delta\right),
\end{aligned}
$$


from which it is apparent that

$$
|(\alpha \boldsymbol{\xi}, \boldsymbol{\delta})| \leqslant Q\left\{\|\eta\|_{-s}+\left(\|\eta\|_{0}+\|\xi\|_{0}\right) h^{s}\right\}\|\psi\|_{s}
$$

for $s \geqslant 1$; (6.12) follows trivially from (6.9) for $s=0$. It should be noted that (6.12) holds when $s=k+1$ as well as for $0 \leqslant s \leqslant k$. Thus,

$$
|(\alpha \boldsymbol{\xi}, \boldsymbol{\delta})| \leqslant \begin{cases}Q\|p\|_{r+1} h^{r+s}\|\psi\|_{s} & \text { for } 0 \leqslant s \leqslant k \text { and } 1 \leqslant r \leqslant k+1 \\ Q\|p\|_{r+2} h^{r+k+1}\|\psi\|_{k+1} & \text { for } s=k+1 \text { and } 0 \leqslant r \leqslant k+1\end{cases}
$$

The inequalities (6.11) and (6.13) can be combined with (6.10) to imply that

$$
\|\xi\|_{-s} \leqslant \begin{cases}Q\|p\|_{r+1} h^{r+s} & \text { for } 0 \leqslant s \leqslant k \text { and } 1 \leqslant r \leqslant k+1 \\ Q\|p\|_{r+2} h^{r+k+1} & \text { for } s=k+1 \text { and } 0 \leqslant r \leqslant k+1\end{cases}
$$

The estimate (6.14) is optimal in order for $0 \leqslant s \leqslant k+1$ and is optimal in regularity for $0 \leqslant s \leqslant k$, with one additional derivative being required of $p$ for $s=k+1$.

Note that we have proved, in particular, that

$$
\left\|p-p_{h}\right\|_{-k-1}+\left\|\mathbf{u}-\mathbf{u}_{h}\right\|_{-k-1}+\left\|\operatorname{div}\left(\mathbf{u}-\mathbf{u}_{h}\right)\right\|_{-k-1} \leqslant Q\|p\|_{k+3} h^{2 k+2}
$$

for $k \geqslant 0$. Since the mixed method is more nearly associated with $L^{2}$-projection than projection into any other Sobolev space $H^{q}(\Omega)$, certainly no greater exponent than $2 k+2$, which is the maximum occurring in $L^{2}$-projection, could be expected. The regularity required to obtain this exponent is correct for the divergence term; a similar observation has been made by Arnold, Douglas, and Gupta for a related mixed method for the equations of plane elasticity.

Our results can be summarized in the following theorem.

THEOREM 6.1. Let $\Omega$ be $(s+2)-r e g u l a r$. Then, for $h$ sufficiently small,

(a) $\left\|p-p_{h}\right\|_{-s}$

$$
\leqslant \begin{cases}Q\|p\|_{r} h^{r+s} & \text { for } 0 \leqslant s \leqslant k-1 \text { and } 2 \leqslant r \leqslant k+1, \\ Q\|p\|_{r+1} h^{r+k} & \text { for } s=k \text { and } 1 \leqslant r \leqslant k+1, \\ Q\|p\|_{r+2} h^{r+k+1} & \text { for } s=k+1 \text { and } 0 \leqslant r \leqslant k+1,\end{cases}
$$

(6.16) (b) $\left\|\mathbf{u}-\mathbf{u}_{h}\right\|_{-s}$

$$
\leqslant \begin{cases}Q\|p\|_{r+1} h^{r+s} & \text { for } 0 \leqslant s \leqslant k \text { and } 1 \leqslant r \leqslant k+1 \\ Q\|p\|_{r+2} h^{r+k+1} & \text { for } s=k+1 \text { and } 0 \leqslant r \leqslant k+1\end{cases}
$$

(c) $\left\|\operatorname{div}\left(\mathbf{u}-\mathbf{u}_{h}\right)\right\|_{-s}$

$$
\leqslant Q\|p\|_{r+2} h^{r+s} \text { for } 0 \leqslant s \leqslant k+1 \text { and } 0 \leqslant r \leqslant k+1 \text {. }
$$

7. The Projection $\Pi_{h}$ on a Boundary Triangle. We shall consider the case of a triangular element $T$ with two straight edges, say $e_{1}$ and $e_{2}$, and one curvilinear edge, $e_{3}$. The rectangular element can be handled similarly. Recall that we have defined the restriction of the Raviart-Thomas spaces to the curvilinear triangle to be the extension of the polynomials that would have been associated with the ordinary triangle with the same vertices. There are five objectives in the definition of $\Pi_{h}$ on 
an ordinary triangle: unisolvence, the reproduction of $\mathbf{P}_{k}(T)$, the orthogonality of $\left(\mathbf{u}-\Pi_{h} \mathbf{u}\right) \cdot v$ to polynomials of degree $k$ on the edges, the orthogonality of $\operatorname{div}\left(\mathbf{u}-\Pi_{h} \mathbf{u}\right)$ to polynomials of degree $k$ on $T$, and the existence of a bound on the map $\Pi_{h}: \mathbf{H}^{1}(T) \rightarrow \mathbf{L}^{2}(T)$. Now, it is a simple exercise to show that the degrees of freedom chosen by Raviart and Thomas are equivalent on an ordinary triangle to the requirement that

$$
\begin{aligned}
& \text { (a) } \int_{e_{i}}\left(\mathbf{u}-\Pi_{h} \mathbf{u}\right) \cdot v \varphi d s=0, \quad \varphi \in P_{k}\left(e_{i}\right), i=1,2,3, \\
& \text { (b) } \int_{T} \operatorname{div}\left(\mathbf{u}-\Pi_{h} \mathbf{u}\right) \psi d x=0, \quad \psi \in P_{k}(T), \\
& \text { (c) } \int_{T}\left(\mathbf{u}-\Pi_{h} \mathbf{u}\right) \cdot \operatorname{curl} \psi d x=0, \quad \psi \in P_{k}(T) .
\end{aligned}
$$

This can be seen perhaps most easily by observing that the following sequence is exact $\left(P_{k}^{\mathbf{C}}\right.$ is the space of polynomials of degree $k$ in one complex variable with complex coefficients):

$$
0 \rightarrow P_{k}^{\mathrm{C}} \stackrel{\subset}{\rightarrow} P_{k} \times P_{k} \stackrel{\text { grad }+ \text { curl }}{\longrightarrow} \mathbf{P}_{k-1} \rightarrow 0 .
$$

We shall select our degrees of freedom from the above list of possibilities augmented by allowing $\psi \in P_{k+1}(T)$ in (7.1c), noting that we do not need to constrain $\Pi_{h} \mathbf{u} \cdot v$ on the curvilinear edge $e_{3}$. It is clear that we can give our degrees of freedom on a reference triangle $\hat{S}=\hat{S}(T)$ having vertices at $(0,0),(0,1)$, and $(1,0)$, where $\hat{S}$ is obtained affinely from $T$ with $e_{1}$ going to $\hat{e}_{1}=((0,0),(0,1))$ and $e_{2}$ to $\hat{e}_{2}=((0,0),(1,0))$. Note that, since $\partial \Omega$ is smooth, the image of $e_{3}$ is smooth and lies in a lense of width $O(h)$ about the segment $((0,1),(1,0))$. First, let us preserve the orthogonalities (7.1b) and those of (7.1a) on $\hat{e}_{1}$ and $\hat{e}_{2}$ :

$$
\begin{array}{ll}
\text { (a) } \int_{\hat{S}} \operatorname{div}(\hat{\mathbf{u}}-\hat{\Pi} \hat{\mathbf{u}}) \hat{\psi} d x=0, & \hat{\psi} \in P_{k}(\hat{S}), \\
\text { (b) } \int_{\hat{e}_{i}}(\hat{\mathbf{u}}-\hat{\Pi} \hat{\mathbf{u}}) \cdot \hat{v} \hat{\varphi} d s=0, & \hat{\varphi} \in P_{k}\left(\hat{e}_{i}\right), i=1,2 .
\end{array}
$$

Let

$$
\hat{\mathbf{Y}}=\left\{\hat{\mathbf{v}} \in \hat{\mathbf{V}}: \hat{\mathbf{v}} \cdot \hat{\mathbf{v}}=0 \text { on } \hat{e}_{1} \text { and } \hat{e}_{2}, \operatorname{div} \hat{\mathbf{v}}=0\right\},
$$

and note that $\hat{\mathbf{Y}}$ is independent of $\hat{S}$ and that, since the conditions $\mathbf{v} \cdot v=0$ on $\hat{e}_{1}$ and $\hat{e}_{2}$ and $\operatorname{div} \hat{\mathbf{v}}=0$ are linearly independent, $\hat{\mathbf{Y}}$ has the correct dimension to provide the required additional degrees of freedom. Now, recall that $\Pi_{h}$ is used solely for analytical purposes and not for computational ones; thus, we can let $\hat{S}^{*}$ be the triangle with vertices $(0,0),\left(0, \frac{1}{2}\right)$, and $\left(\frac{1}{2}, 0\right)$ which lies inside $\hat{S}$ for $h$ even reasonably small, and then impose moments against $\hat{\mathbf{Y}}$ over $\hat{S}^{*}$ :

$$
\int_{\hat{S}^{*}}(\mathbf{u}-\hat{\Pi} \mathbf{u}) \cdot \hat{\mathbf{v}} d x=0, \quad \hat{v} \in \hat{\mathbf{Y}} .
$$

Note that, if $\hat{\mathbf{v}} \in \hat{\mathbf{Y}}, \hat{\mathbf{v}} \in \mathbf{P}_{k}$, since $\operatorname{div} \hat{\mathbf{v}}=0$; this is one of the properties used by Raviart and Thomas to define $\hat{\mathbf{V}}$. 
The space $\hat{\mathbf{Y}}$ is trivially found for small $k$. For $k=0, \hat{Y}$ is the null space. For $k=1, \hat{\mathbf{Y}}$ is one-dimensional and is the span of the vector $(x,-y)$. For $k=2, \hat{\mathbf{Y}}$ has dimension three and

$$
\mathbf{v} \in \hat{\mathbf{Y}} \Leftrightarrow \mathbf{v}=\left[\begin{array}{c}
x(a+b x+2 c y) \\
-y(a+2 b x+c y)
\end{array}\right] .
$$

Note that $\hat{\mathbf{Y}} \subset \operatorname{curl}\left(P_{k+1}(\hat{S})\right)$.

It is clear that $\Pi_{h}$ reduces to the identity on $\hat{V}$. It is also clear that

$$
\|\operatorname{div} \hat{\Pi} \mathbf{u}\|_{0, \hat{s}}+\|\hat{\Pi} \hat{\mathbf{u}} \cdot \hat{\boldsymbol{v}}\|_{0, \hat{e}_{1} \cup \hat{e}_{2}}+\left\|\operatorname{Proj} j_{\hat{Y}} \hat{\Pi} \hat{\mathbf{u}}\right\|_{0, \hat{s}^{*}} \leqslant Q\|\hat{\mathbf{u}}\|_{1, \hat{s}},
$$

which establishes the boundedness of $\hat{\Pi}$ as a map from $\mathbf{H}^{1}(\hat{S})$ to $\mathbf{L}^{2}(\hat{S})$, since the vanishing of the terms on the left-hand side of (7.5) requires חû to vanish. Finally, a version of the Bramble-Hilbert lemma given by Dupont and Scott [3, Theorem 5.1] shows that there exists a constant $Q_{1}$ that is independent of $\hat{S}$ within the set arising herein such that

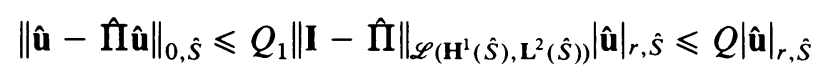

for $1 \leqslant r \leqslant k+1$, where $|\cdot|_{r, s}$ is the seminorm associated with derivatives of exact total order $r$ over the set $\hat{S}$. The usual scaling shows that

$$
\left\|\mathbf{u}-\Pi_{h} \mathbf{u}\right\|_{0, T} \leqslant Q|\mathbf{u}|_{r, T} h^{r}, \quad 1 \leqslant r \leqslant k+1,
$$

where $\Pi_{h}$ is the projection operator on $\mathbf{H}^{1}(T)$ such that

$$
\widehat{\Pi_{h} \mathbf{u}}=\hat{\Pi} \hat{u}, \quad \hat{\Pi}=\hat{\Pi}_{\hat{s}} .
$$

This completes the description of the restriction of $\Pi_{h}$ to a boundary triangle. If $P_{h}$ remains the $L^{2}$-projection onto $P_{k}(T)$ when restricted to $L^{2}(T)$, then the global $\Pi_{h}$-projection of $\mathbf{V}$ onto $\mathbf{V}_{h}$ has the desired properties that were listed in (2.3) and (2.4).

This section represents joint work of Douglas N. Arnold and the authors.

8. The Three Space Variable Problem. Let $\Omega$ be a bounded domain in $\mathbf{R}^{3}$ with smooth boundary $\partial \Omega$. Let $\mathscr{T}_{h}$ be either a simplicial decomposition of $\Omega$ into simplices with maximum diameter $h$ and minimal solid angle at a vertex greater than some positive constant or a decomposition into rectangular elements having maximum diameter $h$. In the simplicial case boundary simplices can have one nonflat face.

The procedure (1.8) remains defined when $\mathbf{V}_{h} \times W_{h}$ are specified following Nedelec, as remarked earlier. Moreover, all of the error estimates in $L^{2}(\Omega)$ and $H^{s}(\Omega)^{\prime}$ remain valid.

Department of Mathematics

University of Chicago

Chicago, Illinois 60637

INRIA

B. P. 105

78153 Le Chesnay, France 
1. F. BREZZI, "On the existence, uniqueness and approximation of saddle-point problems arising from Lagrangian multipliers," RAIRO Anal. Numér., v. 2, 1974, pp. 129-151.

2. J. Douglas, JR. \& J. E. Roberts, "Mixed finite element methods for second order elliptic problems," Mat. Apl. Comput., v. 1, 1982, pp. 91-103.

3. T. Dupont \& R. ScotT, "Polynomial approximation of functions in Sobolev space," Math. Comp., v. 34, 1980, pp. 441-463.

4. R. FALK \& J. OSBORN, "Error estimates for mixed methods," RAIRO Anal. Numér., v. 14, 1980, pp. 249-277.

5. C. JoHnson \& V. ThOMEe, "Error estimates for some mixed finite element methods for parabolic type problems," RAIRO Anal. Numér., v. 15, 1981, pp. 41-78.

6. J. L. Lions \& E. Magenes, Non-Homogeneous Boundary Value Problems and Applications, Vol. I, Springer-Verlag, Berlin, 1970.

7. J. C. NeDElec, “Mixed finite elements in $\mathbf{R}^{3}$," Numer. Math., v. 35, 1980, pp. 315-341.

8. P. A. Raviart \& J. M. Thomas, "A mixed finite element method for 2 nd order elliptic problems," Mathematical Aspects of the Finite Element Method, Lecture Notes in Math., Vol. 606, Springer-Verlag, Berlin, 1977.

9. A. H. Schatz, "An observation concerning Ritz-Galerkin methods with indefinite bilinear forms," Math. Comp., v. 28, 1974, pp. 959-962.

10. R. Scholz, " $L_{\infty}$-convergence of saddle-point approximation for second order problems," $R A I R O$ Anal. Numér., v. 11, 1977, pp. 209-216.

11. R. Scholz, "A remark on the rate of convergence for a mixed finite element method for second order problems,” Numer. Funct. Anal. Optim., v. 4, 1981/1982, pp. 169-177.

12. R. Scholz, “Optimal $L_{\infty}$-estimates for a mixed finite element method for second order elliptic and parabolic problems,” Calcolo, v. 20, 1983, pp. 355-377.

13. J. M. Thomas, Sur l'Analyse Numérique des Méthodes d'Éléments Finis Hybrides et Mixtes, Thèse, Université P. et M. Curie, Paris, 1977. 